\title{
Geochemical and isotopic evidence of groundwater salinization processes in the Essaouira region, north-west coast, Morocco
}

\author{
Otman EL Mountassir ${ }^{1}$ (D) Mohammed Bahir $^{1,2} \cdot$ Driss Ouazar $^{3} \cdot$ Abdelghani Chehbouni $^{2,4} \cdot$ Paula M. Carreira $^{4,5}$
}

Received: 23 November 2020 / Accepted: 3 May 2021

Published online: 11 June 2021

(c) The Author(s) 2021

OPEN

\begin{abstract}
The city of Essaouira is located along the north-west coast of Morocco, where groundwater is the main source of drinking, domestic and agricultural water. In recent decades, the salinity of groundwater has increased, which is why geochemical techniques and environmental isotopes have been used to determine the main sources of groundwater recharge and salinization. The hydrochemical study shows that for the years 1995, 2007, 2016 and 2019, the chemical composition of groundwater in the study area consists of $\mathrm{HCO}_{3}-\mathrm{Ca}-\mathrm{Mg}, \mathrm{Cl}-\mathrm{Ca}-\mathrm{Mg}, \mathrm{SO}_{4}-\mathrm{Ca}$ and $\mathrm{Cl}-\mathrm{Na}$ chemical facies. The results show that from 1995 to 2019, electrical conductivity increased and that could be explained by a decrease in annual rainfall in relation to climate change and water-rock interaction processes. Geochemical and environmental isotope data show that the main geochemical mechanisms controlling the hydrochemical evolution of groundwater in the CenomanianTuronian aquifer are the water-rock interaction and the cation exchange process. The diagram of $\delta^{2} \mathrm{H}=8 * \delta^{18} \mathrm{O}+10$ shows that the isotopic contents are close or above to the Global Meteoric Water Line, which suggests that the aquifer is recharged by precipitation of Atlantic origin. In conclusion, groundwater withdrawal should be well controlled to prevent groundwater salinization and further intrusion of seawater due to the lack of annual groundwater recharge in the Essaouira region.
\end{abstract}

Keywords Stable isotopes · Essaouira · Groundwater · Climate change · Geochemistry

\section{Introduction}

In most of the arid and semiarid regions of the world, the availability of sufficient freshwater has become a factor limiting growth [1-3]. In parts of North Africa, where water scarcity has always been a dominant problem, interference with the natural hydrological cycle through overexploitation of groundwater resources and changes in land use has caused not only reduction of available water, but also degradation of water quality [4-6].
The geochemistry of groundwater in coastal aquifers represents previous geological conditions and current anthropogenic processes that affect the quality of groundwater [7-10]. However, the local and regional meaning of these aquifers, the key hydrogeological trends, hydrochemical characteristics and groundwater origins remain poorly known [11-13]. The combination of geochemistry and isotopic tools can lead to relevant information on the origin of mineralization of karst water [14-17]. Stable isotopes integrated with major ions in groundwater

$\triangle$ Otman EL Mountassir, otman.elmountassir@ced.uca.ma| ${ }^{1}$ High Energy and Astrophysics Laboratory, Faculty of Sciences Semlalia, Cadi Ayyad University, Marrakech, Morocco. ${ }^{2}$ International Water Research Institute (IWRI), Mohammed VI Polytechnic University (UM6P), Benguerir, Morocco. ${ }^{3}$ Mohammadia School of Engineers, Mohammed V University, Rabat, Morocco. ${ }^{4}$ Institut de Recherche Pour Le Développement (IRD), Unité Mixte de Recherche (UMR), Centre D'études Spatiales de La Biosphère (CESBIO), Université de Toulouse, Toulouse, France. ${ }^{5}$ Centro de Ciências e Tecnologias Nucleares, C2TN/IST, Universidade de Lisboa, Lisbon, Portugal. 
have been employed in regional groundwater studies at different locations across the world. The works of [18-24] have demonstrated effectiveness of this approach in understanding recharge pattern, origin and residence time of groundwater from specific sites to basin-scale hydrogeological investigations. Most of these studies have contributed vital information and knowledge that are useful in global groundwater resource management. Dissolved major ions and stable environmental isotopes $\left(\delta^{18} \mathrm{O}\right.$ and $\left.\delta^{2} \mathrm{H}\right)$ have been used to describe the sources of salinization and hydrogeochemical processes affecting groundwater quality in this complex system [25-27]. In addition, the objective of this research is to determine the main source(s) of groundwater mineralization in different aquifers (Plio-Quaternary and Cenomanian-Turonian). The goal of the current study system is to provide baseline information on: (1) the hydrochemical characteristics of groundwater, (2) the key processes that deteriorate the quality of groundwater and (3) the sources of groundwater recharge and their mixing pathways.

\section{Climate}

The Meskala-Ouazzi sub-basin is located in the northeastern part of Essaouira city (Fig. 1). It is bordered on the north by Jbel Hadid, on the south by Igrounzar Wadi, on the east by the Bouabout region and on the west by
Fig. 1 Study area and locations of groundwater samples

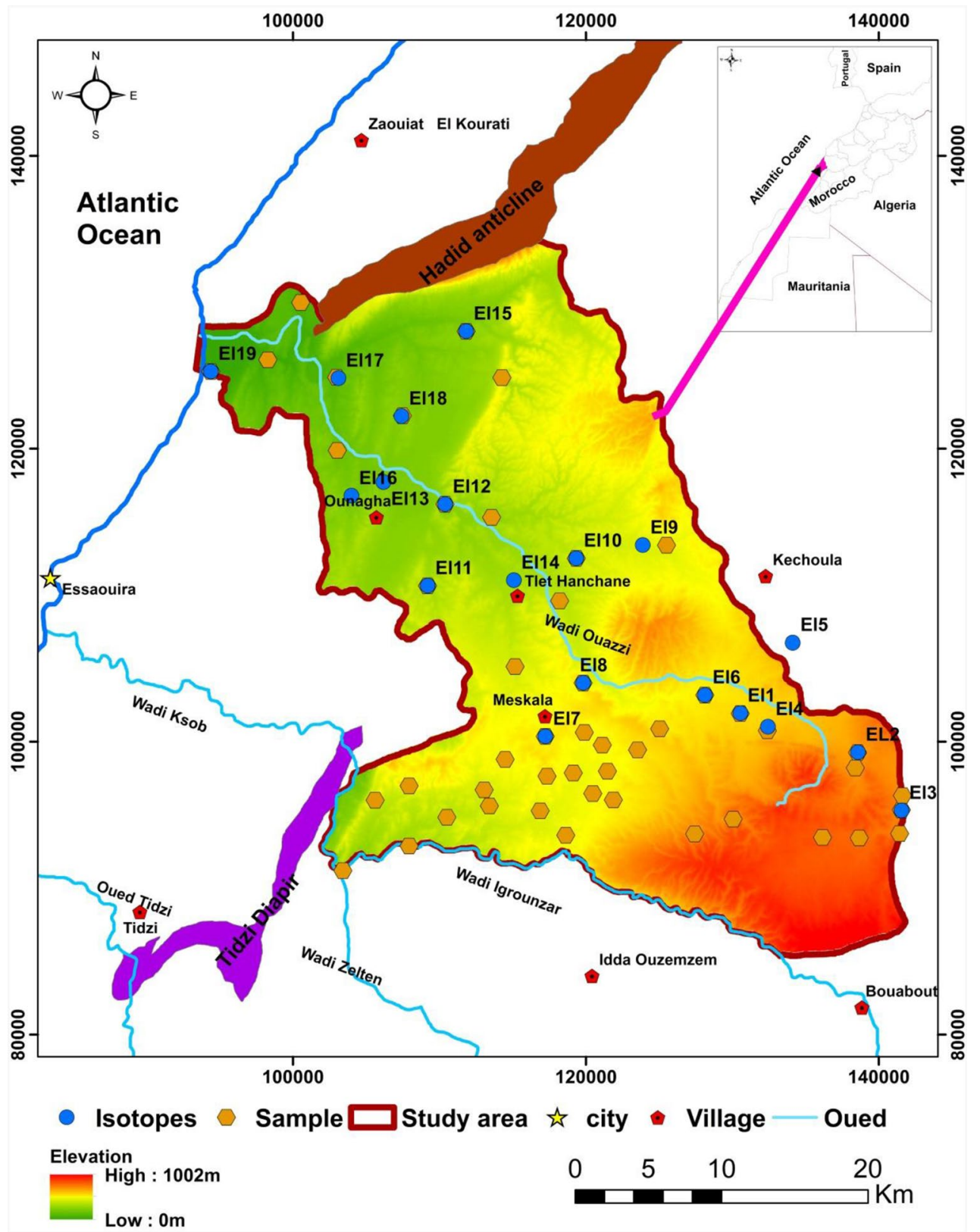


the Atlantic Ocean (Fig. 1). This study is crossed by Wadi Ouazzi and Wadi Igrounzar whose natural outlet is the Atlantic Ocean and has a total area of $1260 \mathrm{~km}^{2}$. The basin is under semiarid climate with irregular rainfall of about $300 \mathrm{~mm}$ year $^{-1}$ and the temperature oscillates around $20^{\circ} \mathrm{C}[9,22]$.

\section{Geological and hydrogeological settings}

Geologically, the upstream part of the study area (Fig. 2) is dominated by the middle and upper Cretaceous outcrop (Albian, Vraconian, Cenomanian and Turonian). These formations consist of banks of limestone and dolomitic intercalated with marls and sandstones. The Albian and Vraconian are represented by green marls (thickness $160 \mathrm{~m}$ ) and dolomitic limestones (thickness $140 \mathrm{~m}$ ). The Cenomanian is characterized by an alternation of grey marls with anhydrite, lumachellic limestones and sandstones in small proportions (thickness $200 \mathrm{~m}$ ) $[28,29]$. The Turonian consists of limestones where silica is very abundant. The downstream part of the study area is characterized by the outcrop of the Plio-Quaternary formations. These are represented by conglomerates, alluviums, colluvium and sandstones (Fig. 2).

Hydrogeologically, two major aquifer systems have been identified: the first is a multi-layered aquifer consisting primarily of Plio-Quaternary detrital deposits (sandstones, conglomerates and sands), responsible for much of the population's water supply. This comes into close contact with the formations of the Triassic and Cretaceous below. The second is the calco-dolomitic layers of the Cenomanian-Turonian age $[28,29]$, reflecting the karstic aquifer; according to $[28,29]$, the Turonian horizon flourishes between 400 and $700 \mathrm{~m}$ altitude (a.s.l.) on the Jbel Kchoula, while the Plio-Quaternary layers grow between sea level and $300 \mathrm{~m}$ altitude (a.s.l.). Transmissivity levels are approximately $4.5 \times 10^{-5}$ to $6 \times 10^{-2} \mathrm{~m}^{2} / \mathrm{s}$ from pumping experiments conducted inside the Plio-Quaternary aquifer [28], while the hydraulic has a permeability of $3.2 \times 10^{-2} \mathrm{~m} / \mathrm{s}$ in the downstream part of the study area [29].

\section{Methodology}

Four water sampling campaigns ( 112 boreholes, wells and springs) were performed in 1995 (18 samples), 2007 (25 samples), 2016 (19 samples) and 2019 (50 samples) in the Meskala-Ouazzi region of Essaouira basin (Fig. 1). Measurements of physico-chemical parameters (temperature,
$\mathrm{pH}$, electrical conductivity and TDS) were taken in the field using the multi-parameter HI9828. The depth to water in each well was measured using a sound piezometric probe. The water samples were taken after pumping for 10-15 min to obtain representative values under ambient aquifer conditions. Samples were collected in clean polyethylene bottle of $500 \mathrm{~mL}$ and then stored at a temperature below $5^{\circ} \mathrm{C}$ before analysis in the laboratory.

Chemical analyses were carried out at the Laboratory of Geosciences and Environment of the Ecole Normale Superieure of Marrakech (Morocco). The contents of chloride $\left(\mathrm{Cl}^{-}\right)$and sulphate $\left(\mathrm{SO}_{4}{ }^{2-}\right)$ anions were determined to adopt the Mohr technique and the nephelometric technique, respectively. $\mathrm{Ca}^{2+}$ and $\mathrm{Mg}^{2+}$ concentrations were determined using the EDTA titrimetric method. $\mathrm{HCO}_{3}{ }^{-}$and $\mathrm{CO}_{3}{ }^{-}$contents were analysed by titration using $0.1 \mathrm{M} \mathrm{HCl}$ acid. $\mathrm{Na}^{+}$and $\mathrm{K}^{+}$were measured by flame spectrometry at the University Center for Analysis, Technology Transfer \& Incubation Expertise (CUAE2TI) at the Faculty of Sciences (Ibn Tofail University, Kenitra, Morocco). The ionic balance for all samples was within $\pm 10 \%$.

Stable isotope levels were measured by laser spectroscopy. The measurements are expressed in per mille compared to the international Standard Mean Ocean Water (SMOW) with an analytical uncertainty of $\pm 0.1 \%$ o for $\delta^{18} \mathrm{O}$ and $\pm 1 \%$ ofor $\delta^{2} \mathrm{H}$. The analyses of stable isotopes $\left(\delta^{2} \mathrm{H}\right.$ and $\delta^{18} \mathrm{O}$ ) were carried out at Centro de Ciências e Tecnologias Nucleares laboratory (CTN/IST, Universidade de Lisboa).

The methodology adopted thus makes it possible to explore the potentials of a multidisciplinary approach, centred on the use of stable isotopes, to determine the recharge areas of the main sources studied. The technique of interpolation (IDW) via a geographical information system (GIS) was used to compile spatiotemporal distribution maps of electrical conductivity.

\section{Results and discussion}

\subsection{Piezometry}

Four piezometers (a-d) were used to track the Meskala-Ouazzi sub-basin piezometric level. Furthermore, Fig. 3 shows that heavy rains cause a continuous increase in the piezometric level, indicating that groundwater levels are highly dependent on precipitation. The four piezometers studied display a general downward trend, despite a small increase in the piezometric level with intense precipitation [4]. Like other basins in coastal areas [30], the Essaouira basin has not been spared the effect of climate change, which is added to the effect of the overexploitation of groundwater. This effect is manifested by (a) an 


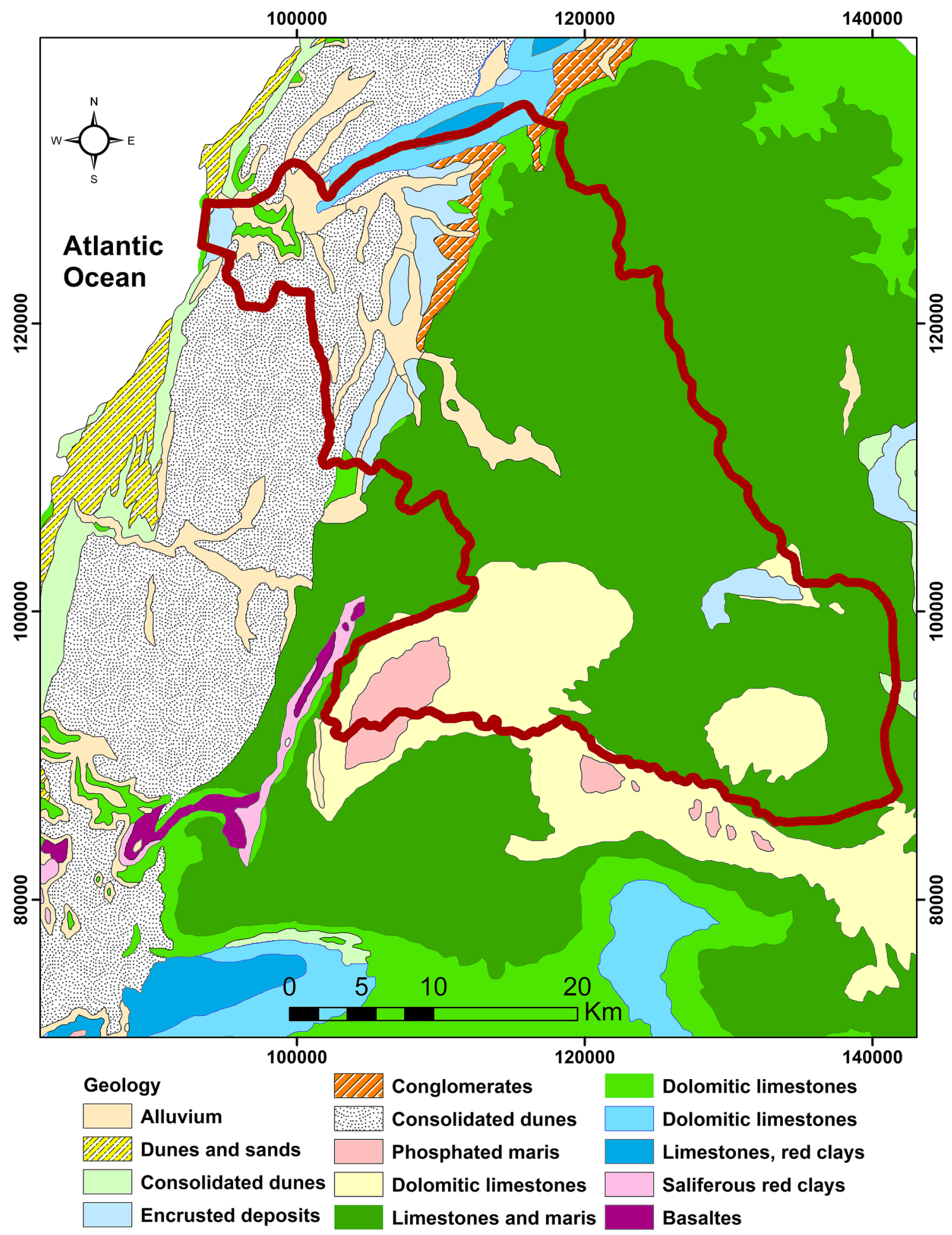

Fig. 2 Geology map in the study area

increase in population of $9 \%$ leading to an increase in irrigated land (1300 ha), (b) upward trend in temperatures with $1.5^{\circ} \mathrm{C}$ warming and a general downward trend in precipitation of $12 \%$ and (c) a deterioration in the quality of groundwater with an increase in salinity. This depletion is due to marine intrusion and a decline in aquifer recharge rates due to the drop in precipitation result of climate change $[4,17]$. 


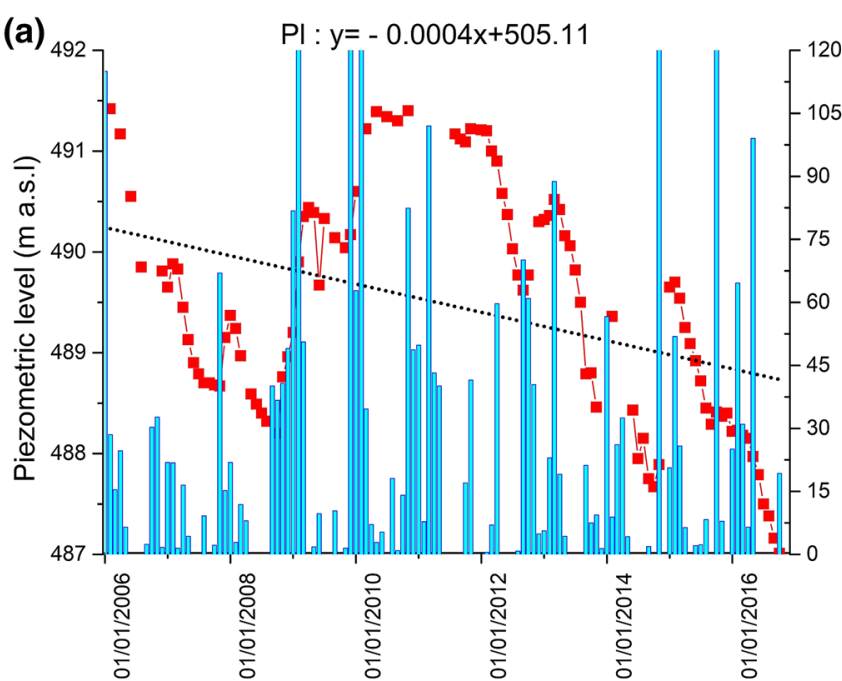

(c)

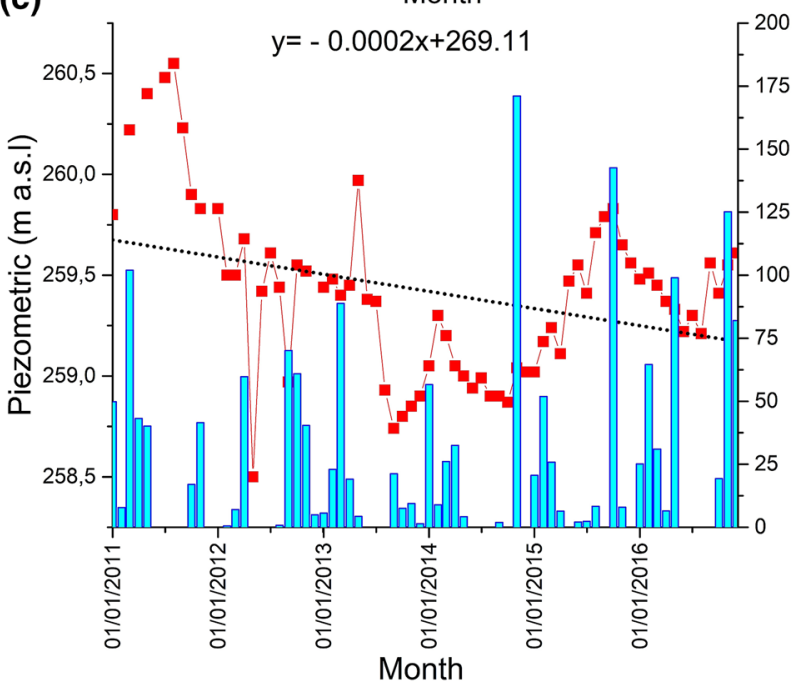

Fig. 3 Piezometric levels for the period 2006-2016 in the study area

\subsection{Hydrochemistry}

The results of chemical analyses are plotted on the Piper diagram [31] (Fig. 4a). This diagram shows that there are four chemical facies in the groundwater of the Meskala-Ouazzi: $\mathrm{HCO}_{3}-\mathrm{Ca}-\mathrm{Mg}, \mathrm{Cl}-\mathrm{Na}, \mathrm{Cl}-\mathrm{Ca}-\mathrm{Mg}$ and $\mathrm{SO}_{4}-\mathrm{Ca}$, with domination of the $\mathrm{Cl}-\mathrm{Ca}-\mathrm{Mg}$ water type. This transition from one facies to another illustrates the importance of the hydrogeochemical processes that control the salinity of the aquifer's waters. For 24 years, the groundwater in the study area retains the same facies.

The $\mathrm{Ca}^{2+}+\mathrm{Mg}^{2+}$ versus $\mathrm{SO}_{4}{ }^{2-}+\mathrm{HCO}_{3}{ }^{-}$plot (Fig. 4b) shows that the cation exchange is not the only process controlling the composition of groundwater. The diagram (Fig. 4b) can be divided into three groups. Samples fall along the 1:1 equiline showing the dissolution of gypsum, calcite and dolomite [32]. The second group

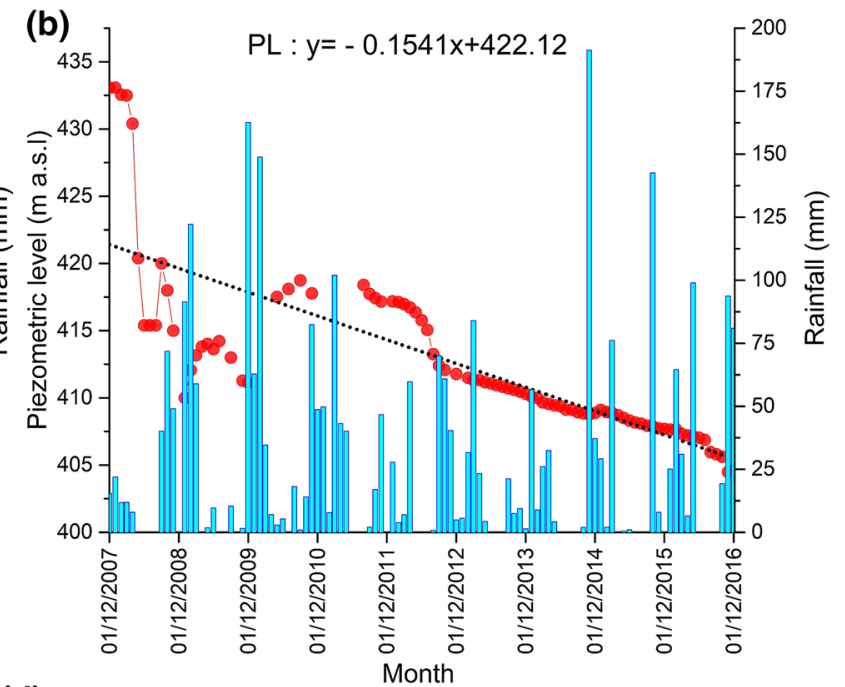

(d)

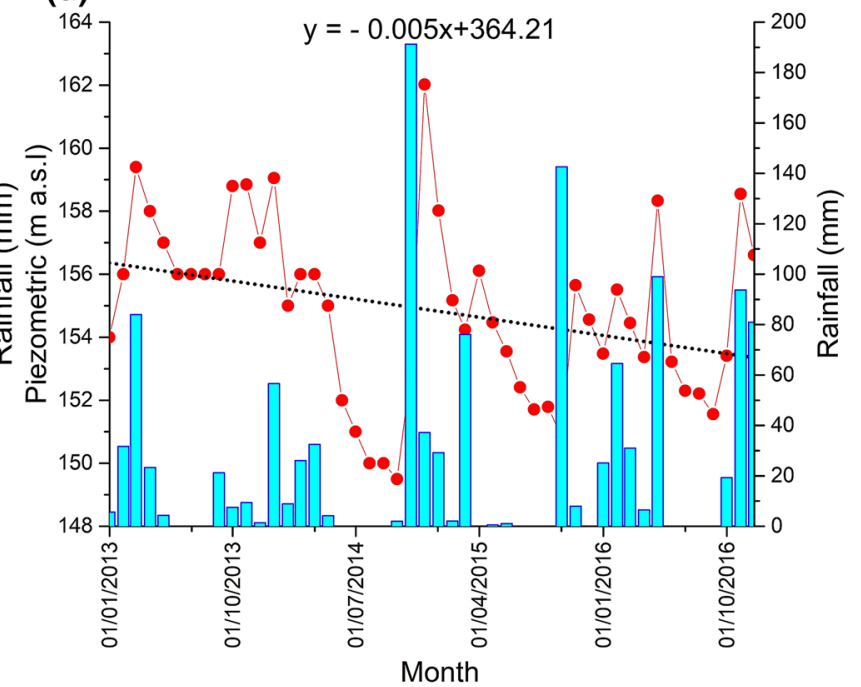

is represented by samples showing slight dominance of $\mathrm{Ca}^{2+}+\mathrm{Mg}^{2+}$ over $\mathrm{HCO}_{3}{ }^{-}+\mathrm{SO}_{4}{ }^{2-}$, and the third group is represented by samples showing a marked increase in the concentration of $\mathrm{Ca}^{2+}+\mathrm{Mg}^{2+}$. The high concentration of $\mathrm{Ca}^{2+}+\mathrm{Mg}^{2+}$ relative to $\mathrm{SO}_{4}{ }^{2-}+\mathrm{HCO}_{3}{ }^{-}$can be a result of reverse ion exchange [30].

Similarly, increasing calcium and bicarbonate levels in the groundwater are considered to be responsible for the dissolution of carbonate minerals. This suggests that reverse ion exchange reactions and weathering are the dominant processes controlling the groundwater chemistry due to the excess $\mathrm{HCO}_{3}{ }^{-}$[33], and this is confirmed by Fig. 8 .

To determine the major elements that contribute to water mineralization, a correlation between major element content and EC was established (Fig. 5). For the groundwater from Meskala-Ouazzi sub-basin, $\mathrm{Ca}^{2+}$, 

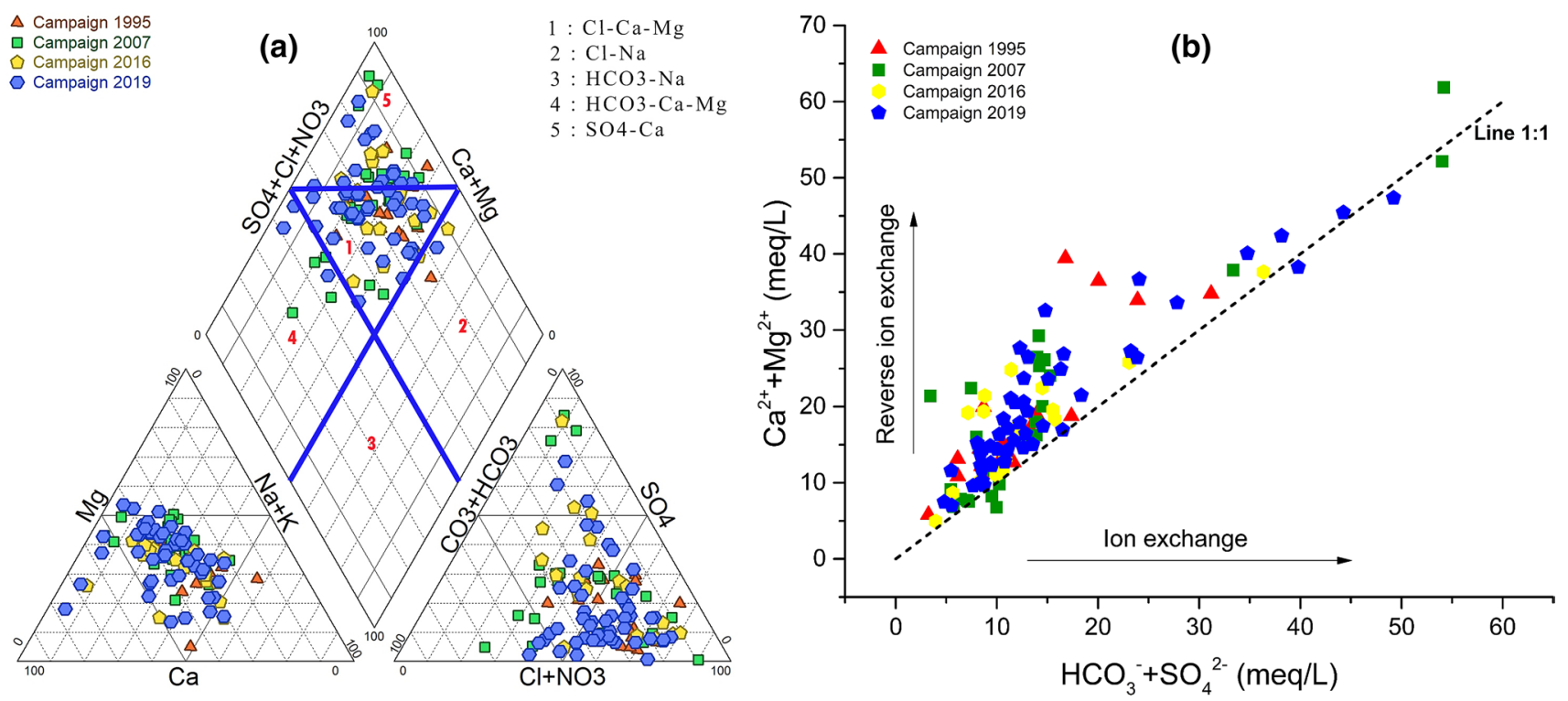

Fig. 4 Hydrochemistry of the four campaigns 1995, 2007, 2016 and 2019 in the study area. a Piper diagram; b correlation between $\mathrm{Ca}^{2+}+\mathrm{Mg}^{2+}(\mathrm{meq} / \mathrm{L})$ and $\mathrm{HCO}_{3}{ }^{-}+\mathrm{SO}_{4}{ }^{2-}(\mathrm{meq} / \mathrm{L})$

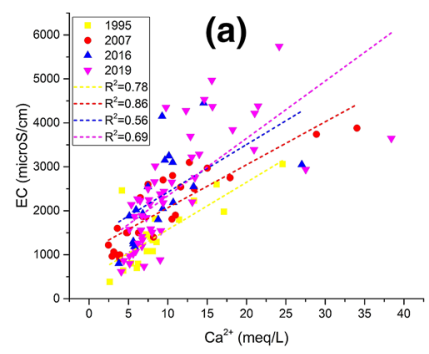

(e)

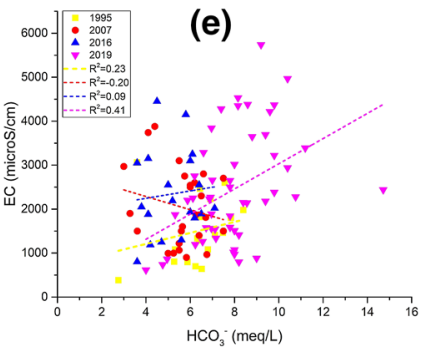

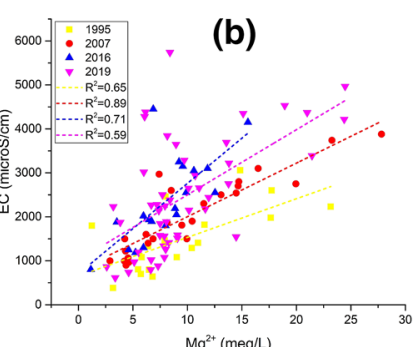

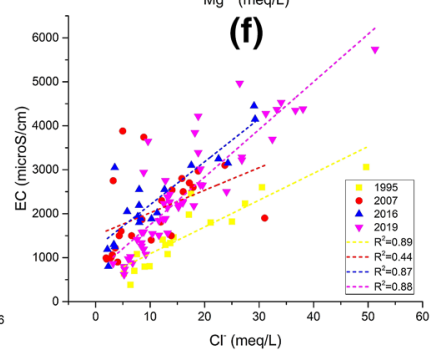

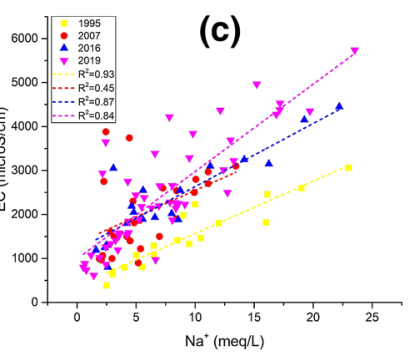

(g)

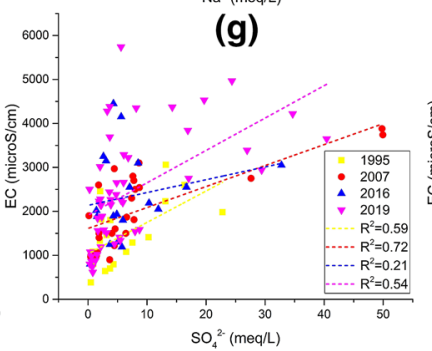

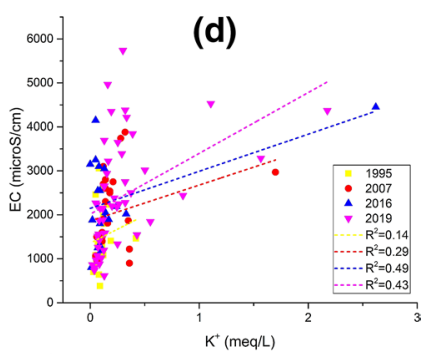

(h)

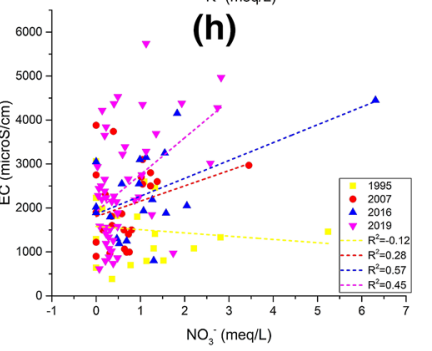

Fig. 5 Bivariate plots of electrical conductivity $(\mathrm{EC})$ plotted against chemical constituents of groundwater

$\mathrm{Mg}^{2+}, \mathrm{SO}_{4}{ }^{2-}$ and $\mathrm{Cl}^{-}$were found to be the main contributors to groundwater mineralization. For the groundwater from the study area, the main elements responsible for groundwater mineralization were $\mathrm{Mg}^{2+}, \mathrm{SO}_{4}{ }^{2-}, \mathrm{Na}^{+}$and $\mathrm{HCO}_{3}{ }^{-}[6,21]$.

The diagram of $\mathrm{Ca}^{2+}$ vs $\mathrm{Mg}^{2+}$ (Fig. 6a) indicates that most points are aligned on the straight line of slope 1 . This reflects the contribution of dolomite dissolution to the mineralization of groundwater, especially in waters sampled in 2016. If the samples plot close to the 1:1 line, calcite, dolomite and gypsum dissolution is prevailing in the aquifer. If the samples fall below the 1:1 line, it reflects the dominance of ion exchange reactions, but if they plot above the 1:1 line, then there is the presence of reverse ion exchange reactions [32].

The $\mathrm{Na}^{+}$vs $\mathrm{Cl}^{-}$diagram (Fig. 6b) indicates that most samples are aligned or before on the 1:1 axis, and the concentration of $\mathrm{Cl}^{-}$is correlated with $\mathrm{Na}^{+}$with a strong correlation $R^{2}=0.9$, indicating that halite dissolution may be the major reaction influencing the water 

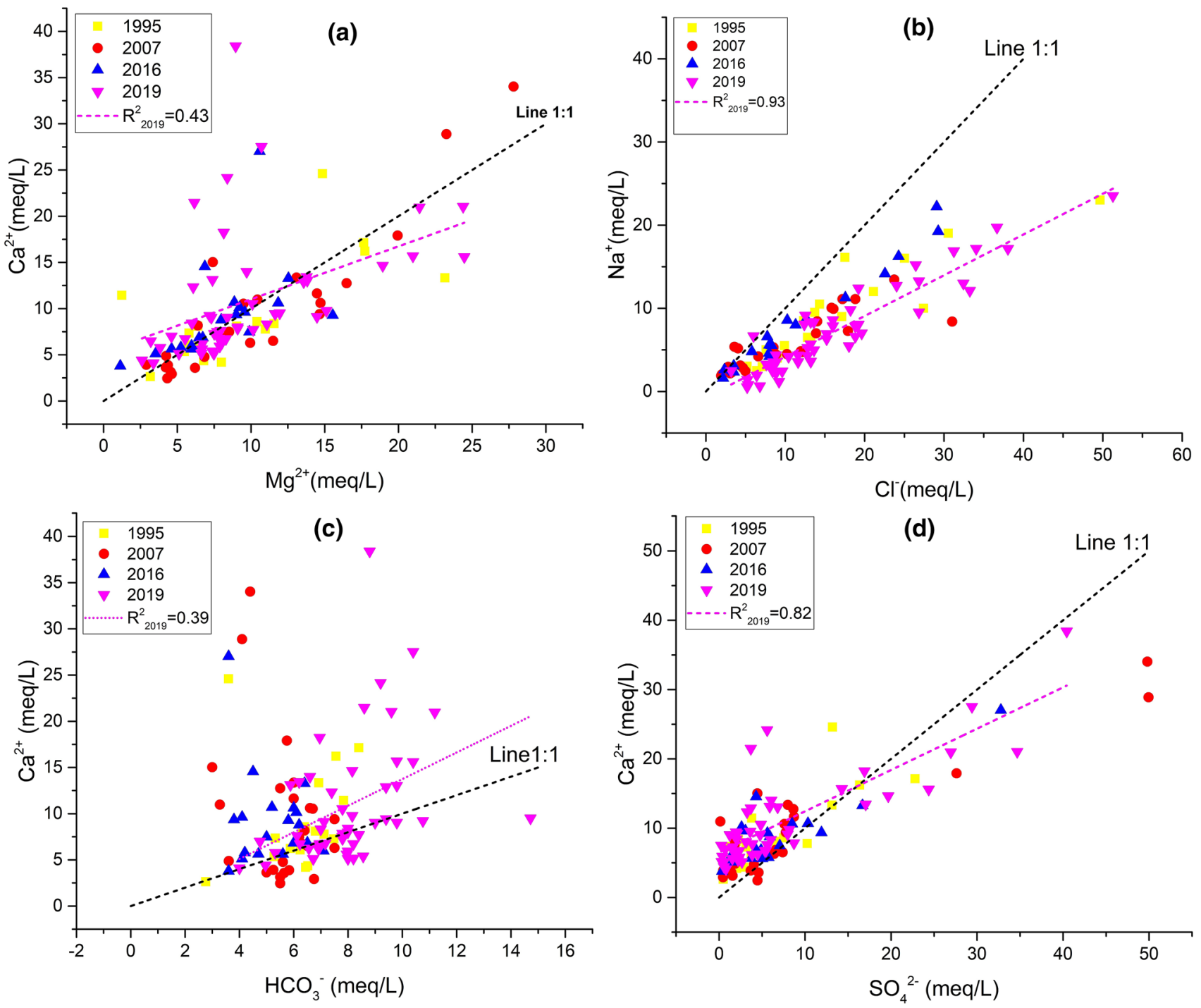

Fig. 6 Correlation diagram: a Ca ${ }^{2+}$ vs $\mathrm{Mg}^{2+}, \mathbf{b ~ N a}{ }^{+} \mathrm{vs} \mathrm{Cl}^{-}, \mathbf{c ~ C a}^{2+} \mathrm{vs}^{-\mathrm{HCO}_{3}}{ }^{-}, \mathbf{d ~ C a}{ }^{2+}$ vs $\mathrm{SO}_{4}{ }^{2}$

chemistry; high values of $\mathrm{Cl}^{-}$and $\mathrm{Na}^{+}$can be resulted from evaporation and the seawater intrusion in groundwater in coastal areas. The water sample points with low densities of $\mathrm{Na}^{+}$and $\mathrm{Cl}^{-}$are basically distributed near the $1: 1$ line under the action of atmospheric precipitation and rock-water interactions. When entering the discharge area (downstream area), the groundwater is gradually affected by surface water recharging, human activities and evaporation. Both $\mathrm{Na}^{+}$and $\mathrm{Cl}^{-}$begin to increase and the distribution of the groundwater sample points along $y=x$ also begins to show a trend of unevenness [34]. This is also confirmed by the plot shown in Fig. 8. No relation had been mentioned between $\mathrm{Ca}^{2+}$ and $\mathrm{HCO}_{3}{ }^{-}$. For the four campaigns (1995, 2007, 2016 and 2019), the interaction between $\mathrm{Ca}^{2+}$ and $\mathrm{HCO}_{3}{ }^{-}$(Fig. $6 \mathrm{C}$ ) is poor. This illustrates the negligible involvement of calcite dissolution in groundwater mineralization in the study area.

On a plot of $\mathrm{Ca}^{2+}$ and $\mathrm{SO}_{4}{ }^{2-}$ (Fig. 6d), majority of the samples for the four campaigns (1995, 2007, 2016 and 2019) plot close to the $1: 1$ line and thus, with high correlation $R^{2}=0.8$, indicate gypsum dissolution. However, the samples that plot below and above the 1:1 line suggest interplay of ion exchange and reverse ion exchange reactions due to excess $\mathrm{SO}_{4}{ }^{2-}$ and $\mathrm{Ca}^{2+}$, respectively (Fig. $6 \mathrm{~d}$ ).

\subsection{Spatiotemporal evolution of electrical conductivity}

Electrical conductivity (EC) values range from 615 to $5738 \mu \mathrm{S} / \mathrm{cm}$ with an average of $2449 \mu \mathrm{S} / \mathrm{cm}$ for 2019 samples, 803 to $4450 \mu \mathrm{S} / \mathrm{cm}$ with an average of $2345 \mu \mathrm{S} /$ 
Table 1 Chemical composition of analysed samples of four campaigns 1995, 2007, 2016 and 2019

\begin{tabular}{|c|c|c|c|c|c|c|c|c|c|c|c|c|}
\hline Sample & $\mathrm{pH}$ & $\begin{array}{l}\mathrm{T} \\
{ }^{\circ} \mathrm{C}\end{array}$ & $\begin{array}{l}\text { EC } \\
\mu S / c m\end{array}$ & $\begin{array}{l}\mathrm{Ca}^{2+} \\
\mathrm{meq} / \mathrm{L}\end{array}$ & $\mathrm{Mg}^{2+}$ & $\mathrm{Na}^{+}$ & $\mathrm{K}^{+}$ & $\mathrm{HCO}_{3}^{-}$ & $\mathrm{Cl}^{-}$ & $\mathrm{SO}_{4}{ }^{2-}$ & $\mathrm{NO}_{3}^{-}$ & $\begin{array}{l}\text { IB } \\
\%\end{array}$ \\
\hline \multicolumn{13}{|c|}{ Campaign 1995} \\
\hline E1 & & 19 & 1980 & 17.13 & 17.65 & 9 & 0.12 & 8.4 & 17.13 & 22.79 & 0.14 & -5 \\
\hline E2 & & 18 & 2600 & 16.21 & 17.73 & 19.01 & 0.12 & 7.56 & 30.54 & 16.35 & 1.09 & -2 \\
\hline E3 & & 17 & 3060 & 24.59 & 14.85 & 23.01 & 0.08 & 3.6 & 49.67 & 13.19 & 0 & -3 \\
\hline E4 & & 19 & 1800 & 11.44 & 1.24 & 12.01 & 0.13 & 7.84 & 21.13 & 3.8 & 0.93 & -15 \\
\hline E5 & & 20 & 2230 & 13.33 & 23.15 & 10 & 0.15 & 6.92 & 27.43 & 13.13 & 0 & -1 \\
\hline E6 & & 18 & 1820 & 8.38 & 11.57 & 16.01 & 0.1 & 6.44 & 25.04 & 2.25 & 0.47 & 3 \\
\hline E7 & & 20 & 804 & 5.35 & 5.48 & 5.5 & 0.03 & 5.28 & 9.92 & 0.88 & 1.52 & -4 \\
\hline E8 & & 19 & 2460 & 4.19 & 8 & 16.12 & 0.05 & 6.44 & 17.53 & 2.07 & 1.33 & 2 \\
\hline E9 & & 19 & 1330 & 8.09 & 6.4 & 9.5 & 0.07 & 6.32 & 13.72 & 2.01 & 2.81 & -2 \\
\hline E10 & & 19 & 1080 & 7.35 & 5.8 & 6.5 & 0.05 & 5.32 & 13.22 & 0.81 & 1.3 & -2 \\
\hline E11 & & 16 & 382 & 2.63 & 3.18 & 2.5 & 0.09 & 2.76 & 6.41 & 0.49 & 0.36 & -9 \\
\hline E12 & & 20 & 1410 & 7.78 & 10.98 & 8.5 & 0.19 & 7.12 & 12.32 & 10.26 & 1.33 & -6 \\
\hline E13 & & 19.5 & 1460 & 7.27 & 7.92 & 10.5 & 0.42 & 7.48 & 14.32 & 3.15 & 5.24 & -7 \\
\hline E14 & & 18 & 640 & 4.35 & 6.8 & 3.02 & 0.08 & 6.52 & 5.21 & 2.91 & 0 & -1 \\
\hline E15 & & 18 & 1080 & 8.1 & 9.04 & 5 & 0.12 & 6.8 & 7.61 & 6.47 & 2.21 & -2 \\
\hline E16 & & 17.5 & 795 & 6.23 & 6.48 & 4 & 0.04 & 5.88 & 9.01 & 4.32 & 1.14 & -10 \\
\hline E17 & & 18 & 700 & 6.07 & 5.73 & 3 & 0.03 & 6.24 & 7.11 & 3.75 & 0.78 & -9 \\
\hline E18 & & 19 & 1290 & 8.58 & 10.41 & 6.5 & 0.11 & 6.4 & 12.82 & 7.52 & 0 & -2 \\
\hline \multicolumn{13}{|c|}{ Campaign 2007} \\
\hline E19 & 7.28 & 22 & 2600 & 7.49 & 8.52 & 7.27 & 0.14 & 6.2 & 17.9 & 1.79 & 1.38 & -8 \\
\hline E20 & 7.24 & 20 & 1500 & 4.77 & 6.85 & 3.13 & 0.06 & 5.6 & 4.35 & 4.06 & 0.8 & 0 \\
\hline E21 & 7.5 & 17.4 & 998 & 3.92 & 2.9 & 2.96 & 0.08 & 5.25 & 2.82 & 0.49 & 0.75 & 3 \\
\hline E22 & 7.39 & 21.9 & 1065 & 3.14 & 4.56 & 2.17 & 0.05 & 5.5 & 3.07 & 1.57 & 0.64 & -4 \\
\hline E23 & 7.69 & 21.3 & 994 & 3.64 & 4.22 & 1.89 & 0.06 & 5 & 1.93 & 1.42 & 0.69 & 4 \\
\hline E24 & 7.64 & 17.4 & 3880 & 34.03 & 27.81 & 2.44 & 0.32 & 4.4 & 4.98 & 49.81 & 0 & 4 \\
\hline E25 & 7.6 & 18.3 & 1600 & 3.59 & 6.21 & 2.84 & 0.11 & 5.65 & 4.67 & 4.59 & 0.36 & -9 \\
\hline E26 & 7.11 & 21.5 & 2750 & 17.91 & 19.95 & 2.27 & 0.21 & 5.75 & 3.23 & 27.62 & 0 & 5 \\
\hline E27 & 6.9 & 26.6 & 2500 & 13.36 & 13.09 & 9.93 & 0.18 & 6 & 16.08 & 8 & 1.23 & 8 \\
\hline E28 & 6.9 & 22.8 & 2800 & 10.61 & 14.72 & 10.06 & 0.14 & 6.6 & 15.83 & 7.61 & 1.23 & 6 \\
\hline E29 & 6.75 & 22.2 & 3100 & 12.75 & 16.49 & 13.45 & 0.12 & 5.5 & 23.73 & 8.65 & 1.05 & 5 \\
\hline E30 & 6.65 & 24.8 & 2700 & 9.4 & 14.66 & 11.11 & 0.12 & 7.5 & 17.22 & 7.77 & 1.02 & 3 \\
\hline E31 & 7.35 & 24.4 & 2540 & 11.64 & 14.48 & 8.43 & 0.18 & 6 & 14.03 & 8.71 & 1.05 & 8 \\
\hline E32 & 7.35 & 22.2 & 2970 & 15.02 & 7.41 & 11.11 & 1.7 & 3 & 18.83 & 4.46 & 3.45 & 8 \\
\hline E33 & 7.8 & 23.2 & 900 & 3.88 & 4.37 & 5.18 & 0.36 & 5.83 & 4.05 & 3.68 & 0 & 1 \\
\hline E34 & 7.1 & 22.1 & 1900 & 10.97 & 10.44 & 8.4 & 0.12 & 3.28 & 31.03 & 0.16 & 0 & -7 \\
\hline E35 & 7.65 & 26.4 & 3740 & 28.88 & 23.26 & 4.43 & 0.28 & 4.1 & 8.84 & 49.94 & 0.39 & -5 \\
\hline E36 & 7.4 & 25 & 1220 & 2.46 & 4.33 & 5.38 & 0.36 & 5.5 & 3.61 & 4.47 & 0 & -4 \\
\hline E37 & 7 & 26.2 & 2300 & 6.51 & 11.5 & 4.74 & 0.14 & 6.5 & 12.12 & 7.4 & 0.21 & -7 \\
\hline E38 & 7.15 & 24.4 & 966 & 2.92 & 4.63 & 2.06 & 0.06 & 6.75 & 2.03 & 0.47 & 0.31 & 1 \\
\hline E39 & 6.85 & 29.5 & 1810 & 10.53 & 9.49 & 4.84 & 0.16 & 6.7 & 11.97 & 7.8 & 0.35 & -3 \\
\hline E40 & 7.1 & 25.8 & 1400 & 8.18 & 6.39 & 4.49 & 0.11 & 6.4 & 10.21 & 1.87 & 0.73 & 0 \\
\hline E41 & 7.35 & 23.5 & 1500 & 4.87 & 4.25 & 6.98 & 0.09 & 3.61 & 13.88 & 1.86 & 0.15 & -9 \\
\hline E42 & 7.1 & 22.8 & 1500 & 6.29 & 9.95 & 4.21 & 0.11 & 7.5 & 6.61 & 6.41 & 0.63 & -1 \\
\hline E43 & 7.05 & 20 & 1867 & 6.84 & 8.15 & 5.29 & 0.35 & 6.26 & 8.52 & 6.55 & 0.58 & -3 \\
\hline \multicolumn{13}{|c|}{ Campaign 2016} \\
\hline E44 & 7.49 & 19.5 & 2190 & 10.69 & 8.87 & 4.62 & 0.24 & 5.2 & 7.98 & 10.34 & 1.27 & -1 \\
\hline E45 & 7.63 & 21 & 1188 & 5.78 & 5.24 & 1.61 & 0.1 & 4.2 & 2.17 & 5.74 & 0.52 & 0 \\
\hline E46 & 7.37 & 19.2 & 1300 & 5.62 & 5.97 & 2.48 & 0.09 & 5.6 & 3.35 & 5.03 & 0.47 & -1 \\
\hline E47 & 7.23 & 22.1 & 1249 & 5.64 & 4.61 & 2.35 & 0.07 & 4.7 & 3.52 & 3.72 & 0.69 & 0 \\
\hline
\end{tabular}


Table 1 (continued)

\begin{tabular}{|c|c|c|c|c|c|c|c|c|c|c|c|c|}
\hline Sample & $\mathrm{pH}$ & $\begin{array}{l}\mathrm{T} \\
{ }^{\circ} \mathrm{C}\end{array}$ & $\begin{array}{l}\text { EC } \\
\mu S / \mathrm{cm}\end{array}$ & $\begin{array}{l}\mathrm{Ca}^{2+} \\
\mathrm{meq} / \mathrm{L}\end{array}$ & $\mathrm{Mg}^{2+}$ & $\mathrm{Na}^{+}$ & $\mathrm{K}^{+}$ & $\mathrm{HCO}_{3}^{-}$ & $\mathrm{Cl}^{-}$ & $\mathrm{SO}_{4}{ }^{2-}$ & $\mathrm{NO}_{3}{ }^{-}$ & $\begin{array}{l}\text { IB } \\
\%\end{array}$ \\
\hline E48 & 7.31 & 19.8 & 803 & 3.79 & 1.14 & 2.61 & 0.01 & 3.6 & 2.35 & 0.37 & 1.3 & 0 \\
\hline E49 & 7.11 & 18.5 & 2050 & 9.36 & 9 & 4.8 & 0.14 & 3.8 & 5.79 & 11.91 & 2.05 & -1 \\
\hline E50 & 7.23 & 18 & 3250 & 10.14 & 9.23 & 14.16 & 0.05 & 6.1 & 22.56 & 2.61 & 1.55 & 1 \\
\hline E51 & 7.03 & 22.1 & 3050 & 27.03 & 10.59 & 3.08 & 0.13 & 3.6 & 3.52 & 32.77 & 0 & 1 \\
\hline E52 & 6.91 & 23.7 & 1800 & 8.78 & 7.99 & 4.21 & 0.09 & 6.2 & 8.02 & 5.91 & 0.32 & 2 \\
\hline E53 & 6.87 & 20.9 & 1883 & 5.12 & 3.53 & 8.57 & 0.02 & 4.1 & 10.25 & 1.55 & 1.58 & -1 \\
\hline E54 & 6.99 & 23 & 4150 & 9.28 & 15.55 & 19.25 & 0.05 & 5.8 & 29.27 & 5.62 & 1.83 & 2 \\
\hline E55 & 7.15 & 23.3 & 1894 & 6.85 & 6.73 & 5.55 & 0.17 & 6.5 & 8.27 & 4.29 & 0 & 1 \\
\hline E56 & 7.07 & 21.2 & 1933 & 6.82 & 6.48 & 6.59 & 0.13 & 6 & 7.73 & 4.88 & 1.07 & 1 \\
\hline E57 & 6.99 & 23.4 & 2550 & 13.28 & 12.54 & 5.59 & 0.07 & 6.4 & 8.01 & 16.68 & 0.58 & 0 \\
\hline E58 & 7.04 & 22.1 & 2020 & 5.97 & 5.97 & 8.01 & 0.33 & 7.1 & 11.3 & 1.43 & 0 & 1 \\
\hline E59 & 7.33 & 24 & 2550 & 7.48 & 9.89 & 8.73 & 0.09 & 5 & 12.66 & 7.06 & 0.97 & 1 \\
\hline E60 & 6.99 & 23.2 & 3100 & 10.62 & 11.85 & 11.28 & 0.08 & 6 & 17.57 & 8.49 & 0.99 & 1 \\
\hline E61 & 7.32 & 19.6 & 3150 & 9.63 & 9.58 & 16.24 & 0 & 4.1 & 24.28 & 3.06 & 1.13 & 4 \\
\hline E62 & 7.11 & 20.5 & 4450 & 14.55 & 6.87 & 22.21 & 2.62 & 4.5 & 29.08 & 4.31 & 6.31 & 2 \\
\hline \multicolumn{13}{|c|}{ Campaign 2019} \\
\hline E63 & 7.44 & 21.48 & 4530 & 14.64 & 18.93 & 17.18 & 1.11 & 8.16 & 34.05 & 19.67 & 0.49 & -9 \\
\hline E64 & 7.82 & 18.02 & 2249 & 7.60 & 7.94 & 8.59 & 0.26 & 6.12 & 16.01 & 5.59 & 0.97 & -8 \\
\hline E65 & 7.13 & 21.71 & 2179 & 9.42 & 11.64 & 5.47 & 0.25 & 9.40 & 18.03 & 1.98 & 0.87 & -6 \\
\hline E66 & 8.38 & 17.8 & 615 & 4.09 & 3.38 & 1.42 & 0.13 & 4.00 & 5.23 & 0.81 & 0.07 & -6 \\
\hline E67 & 7.71 & 20.75 & 2381 & 9.04 & 8.40 & 5.69 & 0.22 & 9.80 & 13.20 & 4.77 & 0.19 & -9 \\
\hline E68 & 7.45 & 20.18 & 3842 & 18.24 & 8.16 & 9.82 & 0.39 & 6.96 & 18.23 & 16.91 & 0.23 & -7 \\
\hline E69 & 7.55 & 20.27 & 2199 & 13.12 & 7.39 & 6.93 & 0.20 & 5.88 & 15.22 & 5.90 & 0.13 & 1 \\
\hline E70 & 7.2 & 23.63 & 1075 & 5.92 & 7.89 & 1.19 & 0.06 & 8.00 & 9.21 & 0.39 & 0.29 & -9 \\
\hline E71 & 7.21 & 24 & 972 & 5.12 & 5.12 & 6.66 & 0.05 & 8.00 & 6.01 & 0.57 & 1.74 & 2 \\
\hline E72 & 7.51 & 22 & 1888 & 6.72 & 8.07 & 5.25 & 0.12 & 6.96 & 12.82 & 2.34 & 0.48 & -6 \\
\hline E73 & 7.45 & 21.06 & 4380 & 21.47 & 6.16 & 17.16 & 0.32 & 8.60 & 38.05 & 3.69 & 1.94 & -7 \\
\hline E74 & 7.66 & 21.35 & 2500 & 7.50 & 7.71 & 12.74 & 0.37 & 7.80 & 24.03 & 0.26 & 0.11 & -6 \\
\hline E75 & 7.6 & 20.9 & 3016 & 8.40 & 6.00 & 12.41 & 0.50 & 8.00 & 19.23 & 2.04 & 2.58 & -8 \\
\hline E76 & 7.78 & 19.96 & 736 & 6.99 & 4.61 & 0.79 & 0.04 & 4.76 & 5.33 & 0.73 & 0.39 & 5 \\
\hline E77 & 7.55 & 18.91 & 882 & 9.03 & 7.31 & 0.65 & 0.09 & 9.00 & 6.81 & 1.24 & 0.29 & -1 \\
\hline E78 & 7.76 & 20.95 & 796 & 5.16 & 6.64 & 0.54 & 0.04 & 8.20 & 5.21 & 0.32 & 0.21 & -6 \\
\hline E79 & 7.45 & 21.44 & 1510 & 8.00 & 9.09 & 3.54 & 0.07 & 8.00 & 11.62 & 3.14 & 0.31 & -5 \\
\hline E80 & 7.42 & 20.8 & 1559 & 6.60 & 7.88 & 3.61 & 0.08 & 6.96 & 13.22 & 1.68 & 0.19 & -10 \\
\hline E81 & 7.08 & 19.45 & 3690 & 12.88 & 13.57 & 13.01 & 0.13 & 9.40 & 32.45 & 3.69 & 1.35 & -8 \\
\hline E82 & 7.16 & 20.3 & 5738 & 24.16 & 8.40 & 23.51 & 0.30 & 9.20 & 51.27 & 5.59 & 1.13 & -9 \\
\hline E83 & 7.78 & 21.26 & 1020 & 5.60 & 6.67 & 1.92 & 0.10 & 7.96 & 6.41 & 1.55 & 0.35 & -6 \\
\hline E84 & 7.76 & 19.3 & 1336 & 6.78 & 7.83 & 2.84 & 0.25 & 7.16 & 8.41 & 5.47 & 0.28 & -9 \\
\hline E85 & 7.99 & 18 & 2750 & 13.44 & 13.78 & 4.30 & 0.32 & 6.20 & 12.82 & 17.05 & 1.03 & -8 \\
\hline E86 & 7.47 & 21.4 & 1411 & 6.72 & 8.29 & 3.26 & 0.08 & 8.20 & 7.61 & 5.34 & 0.23 & -8 \\
\hline E87 & 7.4 & 19.63 & 1584 & 7.84 & 9.10 & 4.37 & 0.14 & 7.80 & 8.81 & 8.71 & 0.08 & -8 \\
\hline E88 & 7.98 & 14.85 & 1843 & 7.28 & 7.51 & 4.35 & 0.55 & 7.80 & 11.62 & 1.61 & 1.26 & -6 \\
\hline E89 & 7.08 & 16.5 & 1574 & 5.12 & 7.48 & 3.53 & 0.13 & 6.72 & 8.49 & 2.68 & 0.48 & -6 \\
\hline E90 & 7.57 & 20.3 & 1543 & 9.12 & 14.47 & 4.19 & 0.43 & 7.12 & 10.41 & 7.98 & 0.23 & 5 \\
\hline E91 & 7.09 & 17.25 & 3646 & 38.40 & 8.96 & 2.43 & 0.25 & 8.80 & 9.61 & 40.42 & 0.19 & -8 \\
\hline E92 & 7.59 & 18.04 & 3389 & 20.96 & 21.43 & 6.60 & 0.29 & 11.20 & 18.83 & 26.95 & 0.65 & -8 \\
\hline E93 & 7.53 & 17.5 & 1192 & 6.72 & 5.53 & 3.46 & 0.12 & 6.23 & 8.81 & 2.16 & 0.23 & -5 \\
\hline E94 & 7.2 & 22.15 & 1261 & 6.24 & 8.01 & 2.43 & 0.07 & 6.56 & 8.41 & 4.49 & 0.39 & -8 \\
\hline E95 & 7.8 & 21.03 & 862 & 4.40 & 2.61 & 2.45 & 0.02 & 4.96 & 3.19 & 0.58 & 0.48 & 1 \\
\hline E96 & 7.24 & 20.5 & 4965 & 15.60 & 24.47 & 15.21 & 0.16 & 10.40 & 26.44 & 24.38 & 2.82 & -7 \\
\hline
\end{tabular}


Table 1 (continued)

\begin{tabular}{llllllllllllll}
\hline Sample & $\mathrm{pH}$ & $\mathrm{T}$ & $\mathrm{EC}$ & $\mathrm{Ca}^{2+}$ & $\mathrm{Mg}^{2+}$ & $\mathrm{Na}^{+}$ & $\mathrm{K}^{+}$ & $\mathrm{HCO}_{3}{ }^{-}$ & $\mathrm{Cl}^{-}$ & $\mathrm{SO}_{4}{ }^{2-}$ & $\mathrm{NO}_{3}^{-}$ & $\begin{array}{l}\mathrm{IB} \\
\%\end{array}$ \\
\hline E97 & 7.01 & 22.83 & 2939 & 27.52 & 10.72 & 2.16 & 0.15 & 10.40 & 8.81 & 29.40 & 0.03 & -9 \\
E98 & 7.65 & 22.05 & 2440 & 9.51 & 11.95 & 4.98 & 0.85 & 14.72 & 13.62 & 3.63 & 0.06 & -8 \\
E99 & 7.4 & 22.9 & 3220 & 13.04 & 13.81 & 13.27 & 0.17 & 9.80 & 26.84 & 6.81 & 0.60 & -4 \\
E100 & 7.56 & 20.96 & 2230 & 6.48 & 3.20 & 9.13 & 0.06 & 6.87 & 12.53 & 1.89 & 0.23 & -7 \\
E101 & 7.56 & 21.45 & 2266 & 6.16 & 6.68 & 8.32 & 0.05 & 6.92 & 13.60 & 3.86 & 0.40 & -8 \\
E102 & 7.22 & 20.68 & 4277 & 12.32 & 6.08 & 16.88 & 4.33 & 7.40 & 31.24 & 3.26 & 2.74 & -6 \\
E103 & 7.28 & 20.8 & 2657 & 8.32 & 11.08 & 8.06 & 0.13 & 7.00 & 19.23 & 6.02 & 0.73 & -9 \\
E104 & 7.3 & 19.15 & 4216 & 21.04 & 24.39 & 7.82 & 0.33 & 9.60 & 18.83 & 34.67 & 0.14 & -8 \\
E105 & 7.27 & 20.8 & 4349 & 9.77 & 15.11 & 19.72 & 0.19 & 8.16 & 36.67 & 8.16 & 1.05 & -9 \\
E106 & 7.43 & 20.8 & 3286 & 14.00 & 9.71 & 9.52 & 1.57 & 6.60 & 26.84 & 6.08 & 1.13 & -8 \\
E107 & 7.38 & 20.95 & 2140 & 5.38 & 7.38 & 8.37 & 0.11 & 8.56 & 13.20 & 2.22 & 0.46 & -7 \\
E108 & 7.18 & 22.2 & 2277 & 9.22 & 7.20 & 7.87 & 0.32 & 10.76 & 16.01 & 2.10 & 0.05 & -8 \\
E109 & 7.5 & 22.7 & 2148 & 7.72 & 10.14 & 6.31 & 0.09 & 8.40 & 15.22 & 3.88 & 0.35 & -7 \\
E110 & 7.32 & 21.5 & 2650 & 10.51 & 10.10 & 7.00 & 0.13 & 7.80 & 19.63 & 4.85 & 0.19 & -8 \\
E111 & 7.4 & 20.8 & 1873 & 5.76 & 3.84 & 8.07 & 0.08 & 5.32 & 12.40 & 2.34 & 0.48 & -7 \\
E112 & 7.4 & 19.2 & 4370 & 15.68 & 20.99 & 12.13 & 2.18 & 9.80 & 33.25 & 14.28 & 0.40 & -6 \\
\hline
\end{tabular}

$\mathrm{cm}$ for 2016 campaign waters, 900 to $3880 \mu \mathrm{S} / \mathrm{cm}$ with an average of $2044 \mu \mathrm{S} / \mathrm{cm}$ for 2007 campaign waters and 382 to $3060 \mu \mathrm{S} / \mathrm{cm}$ with an average of $1495 \mu \mathrm{S} /$ $\mathrm{cm}$ for 1900 campaign waters (Table 1). However, an increase in EC values has been observed from 1995 to 2019. The spatial distribution of EC values (Fig. 7a-d) for the four campaigns indicates that the salinity of groundwater in the study area where groundwater discharges into the Atlantic Ocean has risen over time, especially in 2019.

\subsection{Mechanisms controlling groundwater chemistry}

The Gibbs diagram is an important tool for identifying these geochemical processes [35]. This consists of a plot of the ratio of dominant cations $\mathrm{Na} /(\mathrm{Na}+\mathrm{Ca})$ and anions $\mathrm{Cl} /\left(\mathrm{Cl}+\mathrm{HCO}_{3}\right)$ against the TDS to determine the general mechanisms controlling the chemical composition of the studied groundwater. Essentially, groundwater chemistry is controlled by three dominant natural mechanisms, including rock weathering dominance, evaporation dominance and precipitation dominance $[36,37]$. As indicated in Fig. 8, the possible causes of cations and anions dominance in groundwater samples are the water-rock interaction and evaporation processes. The dominance of calcium and bicarbonate ions in the groundwater samples is due to weathering process in aquifer rocks. Evaporation greatly increases concentrations of ions formed by chemical weathering, leading to higher salinity.

\subsection{Environmental isotopes $\left(\delta^{2} \mathrm{H}\right.$ and $\left.\delta^{18} \mathrm{O}\right)$}

A total of 19 samples collected in 2016 were analysed for stable isotopes $\left(\delta^{18} \mathrm{O}\right.$ and $\left.\delta^{2} \mathrm{H}\right)$. Seven samples represent the downstream of study area (Plio-Quaternary aquifer), and 12 samples represent the upstream of study area (Cenomanian-Turonian aquifer). For the upstream samples, the oxygen- 18 contents vary between a minimum of $-6.01 \%$ vs SMOW and a maximum of $-3.28 \%$ vs SMOW, with an average of $-5.04 \%$ vs SMOW. For deuterium, the maximum value is $-21.2 \%$ vs SMOW and the minimum value equal to $-34.5 .8 \%$ vs SMOW with an average of $-29.8 \%$ v v SMOW (Table 2). As for the downstream samples, the contents of oxygen-18 vary between a minimum of $-5.08 \%$ vs SMOW and a maximum of $-3.9 \%$ vs SMOW, with an average of $-4.63 \%$ vs SMOW. For deuterium, the maximum value is $-20.2 \%$ vs SMOW and the minimum value equal to $-28.7 \%$ vs SMOW with an average of $-24.87 \%$ vs SMOW (Table 2). The results of isotopic analysis, the global meteoric water line [38] and the local meteoric line [39], were reported on the deuterium-oxygen 18 diagram; this diagram (Fig. 9a) shows that two groups of water can be distinguished.

For the isotopic characterization of study area, two reference lines were used: the global meteoric water line (GMWL) following Eq. (1) [38],

$\delta^{2} \mathrm{H}=8 * \delta^{18} \mathrm{O}+10$.

And the local meteoric water line of Essaouira basin (LMWL) according to Eq. (2) [39]: 

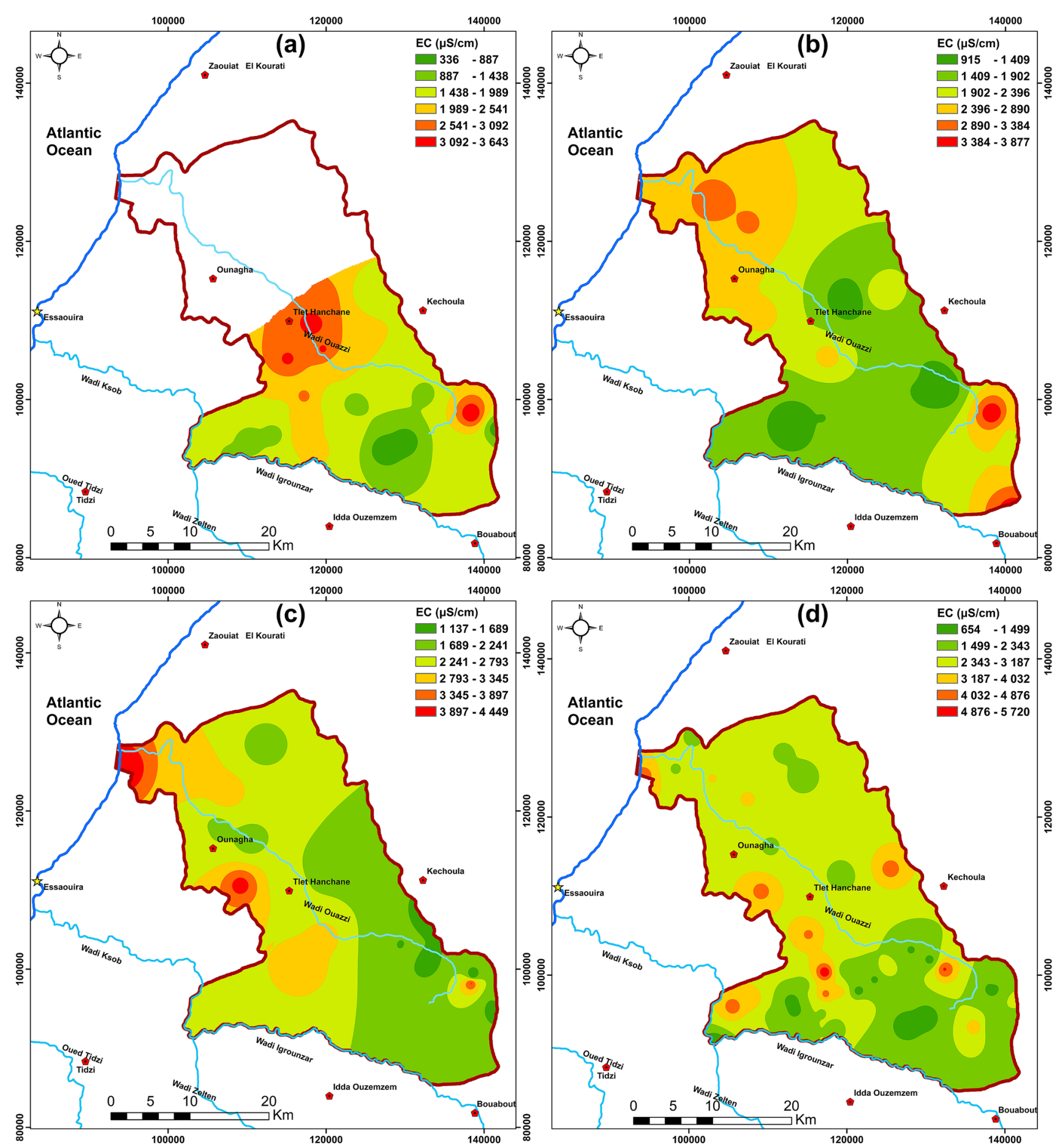

Fig. 7 Spatial distribution of EC in 1995 (a), 2007 (b), 2016 (c) and 2019 (d) 

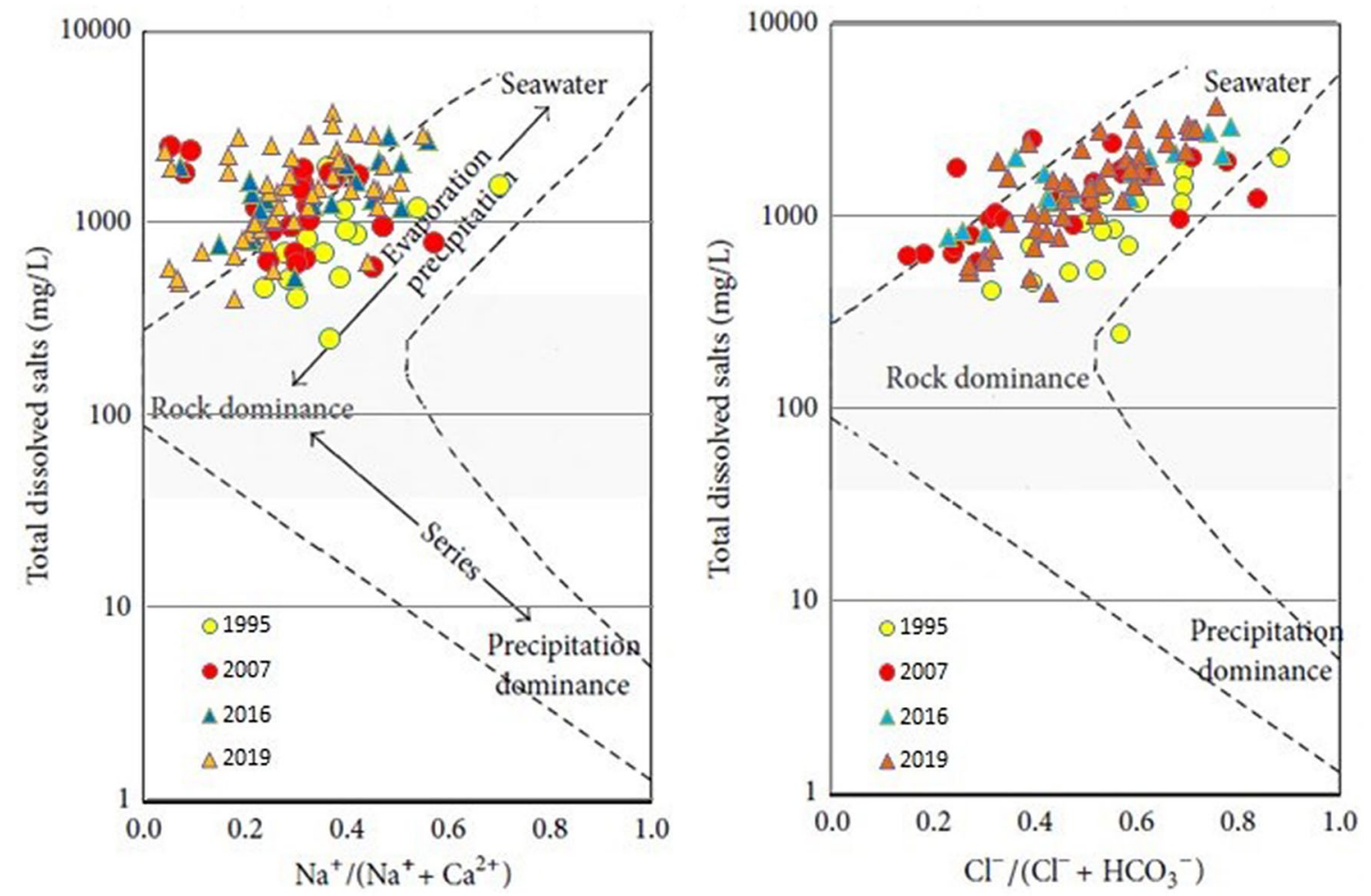

Fig. 8 Gibbs diagrams for the major ion composition of the groundwater in the study area

$\delta^{2} \mathrm{H}=7.95 * \delta^{18} \mathrm{O}+11.3$

The first group is characterized by the most enriched samples, generally from the upstream of study area, that plot below the two reference lines (GMWL and LMWL), as like samples (El 4, El 2, EI 1, El 5, El 7). These water samples are located in the zone of the aquifer recharge, which are mainly characterized by the absence of soil (Fig. 1). This trend could indicate recharge of the aquifer by evaporated meteoric water and/or the return flow of evaporated irrigation water. Evaporation can be produced either before recharging when precipitation passes through the atmosphere with low water content or after recharging by leaching heavy isotopes accumulated during the dry season in the unsaturated zone. The upstream study areas, which are characterized by an altitude above $350 \mathrm{~m}$ a.s.l [40], constitute the beginning of the recharge chain.

The second group represents the majority of samples, situated close to the LMWL and GMWL. These waters come from rapid meteoric water infiltration without any modification of their content isotopic. The values of $\delta^{2} \mathrm{H}$ and $\delta^{18} \mathrm{O}$ on the intersection were the recharge (initial) isotope values from precipitation to the groundwater [41].

The plot of $\mathrm{Cl}^{-}$versus $\delta^{18} \mathrm{O}$ is utilized to identify the dominant mechanism controlling groundwater salinization (Fig. 9b). From the plot of $\mathrm{Cl}^{-}$vs $\delta^{18} \mathrm{O}$, some number of groundwater showed fairly constant $\delta^{18} \mathrm{O}$ with increased chloride concentration. This suggests that dissolution of minerals is significant in this area. Furthermore, the analysis of the $\mathrm{Cl}^{-}$vs $\delta^{18} \mathrm{O}$ relationship (Fig. 9b) shows that the most groundwater samples recorded low chloride concentration with high $\delta^{18} \mathrm{O}$ values. This may suggest that such groundwaters are likely recharged by an enriched source or maybe interacting with some surface water with low chloride and enriched $\delta^{18} \mathrm{O}$.

On this basis, three distinct trends can be observed which reflects the impacts of the mixing process and evaporation on the isotopes and salinity of groundwater (Fig. 9b).

As shown in Fig. 9b, the increase in the values of $\delta^{18} \mathrm{O}$ is greater than that of the concentration of $\mathrm{Cl}^{-}$, indicating a 
Table 2 Isotopic composition of analysed samples campaign 2016 in Meskala-Ouazzi sub-basin

\begin{tabular}{llllll}
\hline Sample & $\mathrm{pH}$ & $\mathrm{T}$ & $\mathrm{EC}$ & $\begin{array}{l}\delta^{2} \mathrm{H} \\
\text { \%o vs. SMOW }\end{array}$ \\
\hline Upstream & & & & & $\delta^{18} \mathrm{O}$ \\
EL1 & 7.2 & 19.5 & 2190 & -31.9 & -4.83 \\
EL2 & 7.8 & 21 & 1188 & -34.4 & -5.16 \\
EL3 & 7.4 & 19.2 & 1300 & -34.1 & -5.73 \\
EL4 & 7.3 & 22.1 & 1249 & -34.5 & -5.52 \\
EL5 & 7.5 & 19.8 & 803 & -28.8 & -4.63 \\
EL6 & 7.7 & 18.5 & 2050 & -28.4 & -4.89 \\
EL7 & 7.5 & 18 & 3250 & -21.2 & -3.28 \\
EL8 & 6.9 & 22.1 & 3050 & -32.9 & -6.01 \\
EL9 & 7.1 & 23.7 & 1800 & -28.8 & -5.5 \\
EL10 & 7.5 & 20.9 & 1883 & -27.6 & -5.06 \\
EL11 & 7.3 & 23 & 4150 & -24.3 & -4.61 \\
EL12 & 7.1 & 23.4 & 2550 & -30.7 & -5.35 \\
Downstream & & & & & \\
EL13 & 7.4 & 23.3 & 1894 & -24.2 & -4.34 \\
EL14 & 7.4 & 21.2 & 1933 & -26.8 & -4.88 \\
EL15 & 7.2 & 22.1 & 2020 & -22.7 & -4.58 \\
EL16 & 7.2 & 24 & 2550 & -28.7 & -5.08 \\
EL17 & 7.1 & 23.2 & 3100 & -27.8 & -5 \\
EL18 & 7.3 & 20.8 & 3150 & -23.7 & -4.63 \\
EL19 & 7.4 & 20.5 & 4450 & -20.2 & -3.9 \\
Min & 6.9 & 18 & 803 & -34.5 & -6.01 \\
Max & 7.8 & 24 & 4450 & -20.2 & -3.28 \\
Mean & 7.33 & 21.38 & 2345.26 & -27.98 & -4.89 \\
Sd & 0.22 & 1.82 & 983.68 & 4.42 & 0.64 \\
\hline & & & & & \\
\hline
\end{tabular}

recharge of groundwater (i.e. irrigation water, river water or precipitation) with a low $\mathrm{Cl}^{-}$the concentration contributes to the enrichment of isotopic values as like in samples $\mathrm{El} 3$ and $\mathrm{El} 4$.

The increase of the $\delta^{18} \mathrm{O}$ values along with $\mathrm{Cl}^{-}$concentrations enrichment could be a result of evaporation (either during recharge, from shallow water levels, or seepage of sewage from the village of Meskala due to the absence of sanitation channels) as like in sample EL7, as the evaporation process enriches both $\delta^{18} \mathrm{O}$ values and $\mathrm{Cl}^{-}$concentrations [42]. The $\delta^{18} \mathrm{O}$ values of groundwater are enriched by surface water recharge (Wadi Igrounzar), while the variation of $\mathrm{Cl}^{-}$concentrations depends on the salinity of the infiltration water that is ultimately attached to the water table. Infiltration water salinity is derived from both the surface water and the soil along the paths [ 43 , 44]. Furthermore, independently of the isotopic exchange, high concentrations of $\mathrm{Cl}^{-}$with low values of $\delta^{18} \mathrm{O}$ are associated with the processes of salt accumulation inside the aquifer (mineral dissolution and transpiration) as in samples El 16 and El 17.

\section{Conclusion}

The socio-economic development in the Essaouira basin depends heavily on groundwater resource; thus, the hydrochemical and isotopic studies made in this article aim to assess the process of salinization and recharge of the Plio-quaternary aquifer and Cenomano-Turonian aquifer given its strategic importance in the study area. Regarding hydrochemistry, with the observed increases in salinity, the chemical composition of groundwater at a given sampling point did not improve. Furthermore, groundwater is mineralized, since EC values are between $1495 \mu \mathrm{S} / \mathrm{cm}$ for 1995 to $2449 \mu \mathrm{S} / \mathrm{cm}$ for 2019 , explained by the decrease in the precipitation due to climate change and overexploitation. The main phenomenon controlling the salinity of the groundwater seems to be the dissolution of evaporites (halite, gypsum and anhydrite) in the study area of Meskala-Ouazzi sub-basin. The results obtained in the isotopic study showed that the groundwater of the Cenemano-Turonian and Plio-Quaternary aquifer comes from precipitation. According to the reports on deuterium and oxygen-18, water quickly enters the aquifer without major change in the relative abundances of these two elements due to the phenomenon of evaporation, except for a few samples which are located in the recharge zone. The combination of chemical and isotopic parameters, in particular $\mathrm{Cl}^{-}$and $\delta^{18} \mathrm{O}$, supports the hydrochemical approach's results by confirming that the dissolution process is one of the key phenomena leading to groundwater mineralization in the study area. Knowledge of sources of groundwater recharge is essential for the protection and management of global aquifer systems, especially in regions where rainfall is likely to be significantly affected by climate change. 

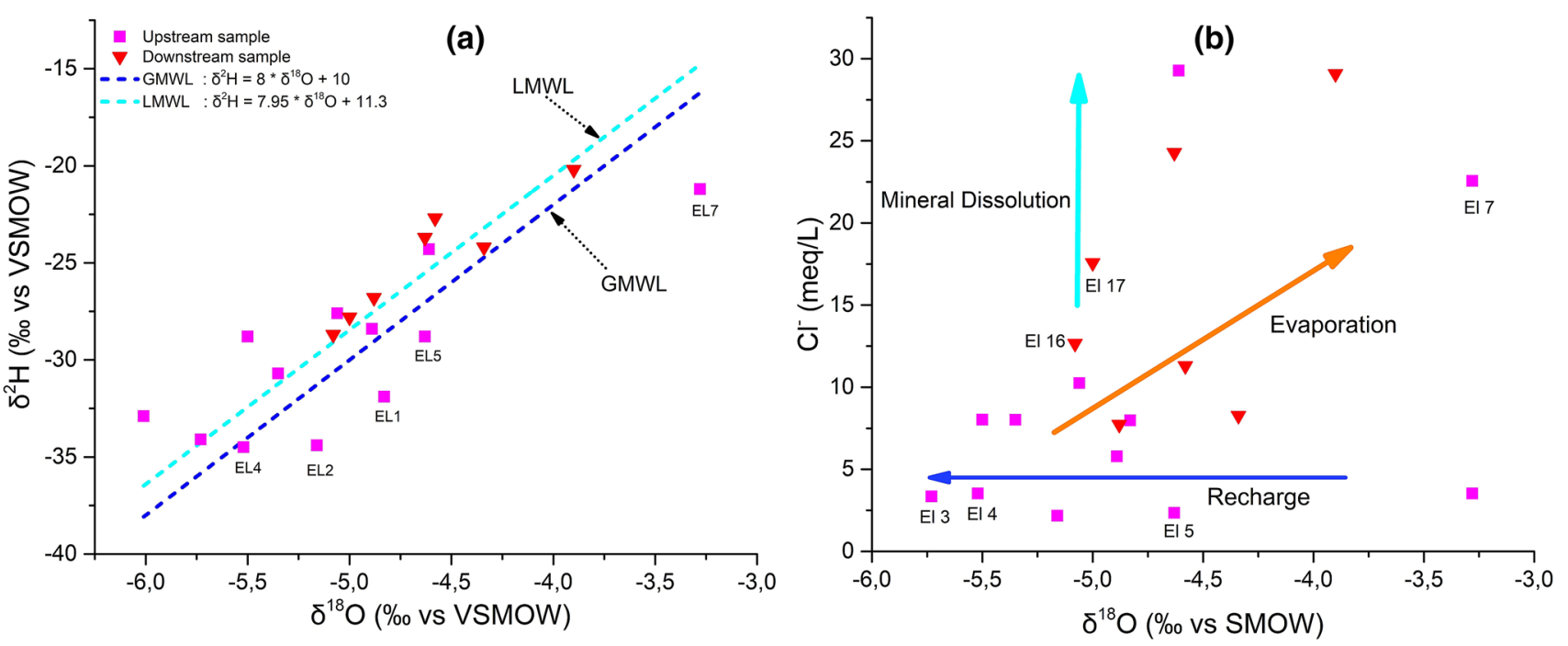

Fig. 9 Isotopic data in the study area. a $\delta^{18} \mathrm{O}$ versus $\delta^{2} \mathrm{H}$ for 2016 campaign; $\mathbf{b} \delta^{18} \mathrm{O}$ versus $\mathrm{Cl}^{-}$diagram

Acknowledgements The authors are grateful to our colleagues for their assistance in data collection and field investigation. Special thanks go to the Editor-in-Chief and the reviewers for their critical reviews and valuable suggestions. We thank the people of Tensift Hydraulic Basin Agency (ABHT) of the city of Marrakech for their help in the data piezometry, especially Mrs. Najar Insaf, Mr. Benaddi Rabia and Mr. Qachar Mohammed. We do not forget to thank the Pr Ebn Touhami Mohamed, dean of the Faculty of Sciences of Kenitra, for his assistance during the chemical analyses of $\mathrm{K}^{+}$and $\mathrm{Na}^{+}$of the groundwater collected in 2019.

\section{Declarations}

Conflict of interest The authors declare that they have no conflict of interest.

Open Access This article is licensed under a Creative Commons Attribution 4.0 International License, which permits use, sharing, adaptation, distribution and reproduction in any medium or format, as long as you give appropriate credit to the original author(s) and the source, provide a link to the Creative Commons licence, and indicate if changes were made. The images or other third party material in this article are included in the article's Creative Commons licence, unless indicated otherwise in a credit line to the material. If material is not included in the article's Creative Commons licence and your intended use is not permitted by statutory regulation or exceeds the permitted use, you will need to obtain permission directly from the copyright holder. To view a copy of this licence, visit http://creativecommons. org/licenses/by/4.0/.

\section{References}

1. Hamed Y, Hadji R, Redhaounia B, Zighmi K, Bâali F, El Gayar A (2018) Climate impact on surface and groundwater in North Africa: a global synthesis of findings and recommendations. Euro-Mediterr J Environ Integr 3:25. https://doi.org/10.1007/ s41207-018-0067-8

\section{SN Applied Sciences}

2. Carreira PM, Marques JM, Nunes D (2014) Source of groundwater salinity in coastline aquifers based on environmental isotopes (Portugal): natural vs. human interference. A Rev Reinterpret Appl Geochem 41:163-175. https://doi.org/10.1016/j.apgeo chem.2013.12.012

3. Bouteraa O, Mebarki A, Bouaicha F, Nouaceur Z, Laignel B (2019) Groundwater quality assessment using multivariate analysis, geostatistical modeling, and water quality index (WQI): a case of study in the Boumerzoug-El Khroub valley of Northeast Algeria. Acta Geochim 38:796-814. https://doi.org/10.1007/ s11631-019-00329-x

4. Bahir M, Ouhamdouch S, Ouazar D, Chehbouni A (2020) Assessment of groundwater quality from semi-arid area for drinking purpose using statistical, water quality index (WQI) and GIS technique. Carbonates Evaporites 35:1-24. https://doi.org/10. 1007/s13146-020-00564-x

5. El Mountassir O, Bahir M, Ouazar D, Carreira PM (2021) Nitrate pollution in groundwater of the Ouazi Basin: case of Essaouira (Southwestern Morocco). In: Abrunhosa M, Chambel A, Peppoloni S, Chaminé HI (eds) Advances in geoethics and groundwater management: theory and practice for a sustainable development. Advances in science, technology and innovation (IEREK interdisciplinary series for sustainable development). Springer, Cham. https://doi.org/10.1007/978-3-030-59320-9_49

6. El Mountassir O, Bahir M, Ouazar D, Carreira PM (2021) For a better understanding of recharge and salinization mechanism of a Cenomanian-Turonian aquifer. In: Abrunhosa M, Chambel A, Peppoloni S, Chaminé $\mathrm{HI}$ (eds) Advances in geoethics and groundwater management: theory and practice for a sustainable development. Advances in science, technology and innovation (IEREK interdisciplinary series for sustainable development). Springer, Cham . https://doi.org/10.1007/978-3-030-59320-9_42

7. Edmunds WM (2003) Renewable and non-renewable groundwater in semi-arid and arid regions. Dev Water Sci 50:265-280

8. Bennetts DA, Webb JA, Stone DJM, Hill DM (2006) Understanding the salinization process for groundwater in an area of southeastern Australia, using hydrochemical and isotopic evidence. J Hydrol 323:178-192. https://doi.org/10.1016/j.jhydrol.2005.08. 023

9. El Mountassir O, Bahir M, Ouazar D, Ouhamdouch S, Chehbouni A, Ouarani M (2020) The use of GIS and water quality index 
to assess groundwater quality of krimat aquifer (Essaouira; Morocco). SN Appl Sci 2:1-16. https://doi.org/10.1007/ s42452-020-2653-Z

10. Ouarani M, Bahir M, Mulla DJ, Ouazar D, Chehbouni A, Dhiba D, Ouhamdouch S, El Mountassir O (2020) Groundwater quality characterization in an overallocated semi-arid coastal area using an integrated approach: case of the Essaouira Basin, Morocco. Water 12:3202. https://doi.org/10.3390/w12113202

11. Kanduč $T$, Šlejkovec $Z$, Vreča $P$, Samardžija Z, Verbovšek T, Božič D, Jamnikar S, Solomon DK, Fernandez DP, Eastoe C, McIntosh J (2019) The effect of geochemical processes on groundwater in the Velenje coal basin, Slovenia: insights from mineralogy, trace elements and isotopes signatures. SN Appl Sci 1:1518. https:// doi.org/10.1007/s42452-019-1561-6

12. Eissa MA, Mahmoud HH, Shouakar-Stash O, El-Shiekh A, Parker B (2016) Geophysical and geochemical studies to delineate seawater intrusion in Bagoush area, Northwestern coast. Egypt J Afr Earth Sci 121:365-381. https://doi.org/10.1016/j.jafrearsci.2016. 05.031

13. Bahir M, Ouhamdouch S (2020) Groundwater quality in semiarid environments (Essaouira Basin, Morocco). Carbonates Evaporites 35:1-16. https://doi.org/10.1007/s13146-020-00576-7

14. Hassen I, Hamzaoui-Azaza F, Bouhlila R (2016) Application of multivariate statistical analysis and hydrochemical and isotopic investigations for evaluation of groundwater quality and its suitability for drinking and agriculture purposes: case of Oum AliThelepte aquifer, central Tunisia. Environ Monit Assess 188:135. https://doi.org/10.1007/s10661-016-5124-7

15. Hamed Y, Dhahri F (2013) Hydro-geochemical and isotopic composition of groundwater, with emphasis on sources of salinity, in the aquifer system in Northwestern Tunisia. J Afr Earth Sci 83:10-24. https://doi.org/10.1016/j.jafrearsci.2013.02.004

16. Hamed Y, Ahmadi R, Demdoum A, Bouri S, Gargouri I, Dhia HB, Al-Gamal S, Laouar R, Choura A (2014) Use of geochemical, isotopic, and age tracer data to develop models of groundwater flow: a case study of Gafsa mining basin-Southern Tunisia. J Afr Earth Sci 100:418-436. https://doi.org/10.1016/j.jafrearsci.2014. 07.012

17. Bahir M, Ouazar D, Goumih A, Ouhamdouch S (2019) Evolution of the chemical and isotopic composition of groundwater under a semi-arid climate; the case of the Cenomano-Turonian aquifer within the Essaouira basin (Morocco). Environ Earth Sci 78:353. https://doi.org/10.1007/s12665-019-8349-2

18. Jassas H, Merkel B (2015) Assessment of hydrochemical evolution of groundwater and its suitability for drinking and irrigation purposes in Al-Khazir Gomal Basin, Northern Iraq. Enviro Earth Sci 74:6647-6663. https://doi.org/10.1007/s12665-015-4664-4

19. Bouaroudj S, Menad A, Bounamous A, Ali-Khodja H, Gherib A, Weigel DE, Chenchouni $\mathrm{H}$ (2019) Assessment of water quality at the largest dam in Algeria (Beni Haroun Dam) and effects of irrigation on soil characteristics of agricultural lands. Chemosphere 219:76-88. https://doi.org/10.1016/j.chemosphere.2018.11.193

20. Bouaicha F, Dib H, Bouteraa O, Manchar N, Boufaa K, Chabour N, Demdoum A (2019) Geochemical assessment, mixing behavior and environmental impact of thermal waters in the Guelma geothermal system, Algeria. Acta Geochim 38:683-702. https:// doi.org/10.1007/s11631-019-00324-2

21. Bahir M, Ouazar D, Ouhamdouch S (2019) Hydrogeochemical investigation and groundwater quality in Essaouira region, Morocco. Mar Freshwater Res 70:1317-1332. https://doi.org/ 10.1071/MF18319

22. Bahir M, Ouhamdouch S, Ouazar D, El Moçayd N (2020) Climate change effect on groundwater characteristics within semi-arid zones from western Morocco. Groundw Sustain Dev 10:1-15. https://doi.org/10.1016/j.gsd.2020.100380
23. Bahir M, El Mountassir O, Ouazar D, Carreira PM (2021) Use of $W Q I$ and isotopes to assess groundwater quality of coastal aquifers (Essaouira, Morocco). In: Abrunhosa M, Chambel A, Peppoloni S, Chaminé HI (eds) Advances in geoethics and groundwater management: theory and practice for a sustainable development. Advances in science, technology and innovation (IEREK interdisciplinary series for sustainable development). Springer, Cham. https://doi.org/10.1007/978-3-030-59320-9_51

24. Bahir M, El Mountassir O, Ouazar D, Carreira PM (2021) Hydrochemical analysis and evaluation of groundwater quality in Ouazi Basin (Essaouira, Morocco). In: Abrunhosa M, Chambel A, Peppoloni S, Chaminé HI (eds) Advances in geoethics and groundwater management : theory and practice for a sustainable development. Advances in science, technology and innovation (IEREK interdisciplinary series for sustainable development). Springer, Cham. https://doi.org/10.1007/978-3-030-59320-9_50

25. Wang Y, Guo Q (2006) Strontium isotope characterization and major iongeochemistry of karst water flow, Shentou, northern China. J Hydrol 328:592-603. https://doi.org/10.1016/j.jhydrol. 2006.01.006

26. Casanova J, Negrel PH, Kloppmann W, Aranyossy JF (2001) Origin of deep saline groundwaters in the Vienne granitoids (France). Geofluids 1:91-101. https://doi.org/10.1046/j.1468-8123.2001. 00009.x

27. Aquilina L, Ladouche B, Doerfliger N, Seidel JL, Bakalowicz M, Dupuy C, LeStrat $P$ (2002) Origin, evolution and residence time of saline thermal fluids (Balaruc springs, southern France): Implications for fluid transfer across the continental shelf. Chem Geol 192:1-21. https://doi.org/10.1016/S0009-2541(02)00160-2

28. Bahir M, Carreira P, Da Silva MO, Fernandes P (2007) Caractérisation hydrodynamique, hydrochimique et isotopique du système aquifère de Kourimat (Bassin d'Essaouira, Maroc). Estud Geol 64:61-73. https://doi.org/10.3989/egeol.08641433

29. Bahir M, Mennani A, Jalal M, Youbi N (2000) Contribution à l'étude des ressources hydriques du bassin synclinal d'Essaouira (Maroc). Estud Geol 56:185-195. https://doi.org/10.3989/egeol. 00563-4150

30. Rajmohan N, Elango L (2004) Identification and evolution of hydrogeochemical processes in the groundwater environment in an area of the Palar and Cheyyar River Basins, Southern India. Environ Geol 46:47-61. https://doi.org/10.1007/ s00254-004-1012-5

31. Piper AM (1944) A graphical interpretation of water analysis. Trans Am Geophys Union 25:914-928

32. Fisher RS, Mullican WF (1997) Hydrochemical evolution of sodium-sulphate and sodium-chloride groundwater beneath the Northern Chihuahuan desert, Trans-Pecos, Texas, USA. Hydrogeol J 5:4-16

33. Singh SP, Tripathi SK, Kumar V, Kumar A, Raha P (2015) Hydrochemical investigation and groundwater quality evaluation for irrigation purpose in some blocks of Varanasi District, Uttar Pradesh, India. Int J Trop Agric 33:1653-1660

34. Zhu G, Wu X, Ge J, Liu F, Zhao W, Wu C (2020) Influence of mining activities on groundwater hydrochemistry and heavy metal migration using a self-organizing map (SOM). J Clean Prod 257:120-664. https://doi.org/10.1016/j.jclepro.2020.120664

35. Gibbs RJ (1970) Mechanisms controlling world water chemistry. Science 170:1088-1090. https://doi.org/10.1126/science.170. 3962.1088

36. Adimalla N, Wu J (2019) Groundwater quality and associated health risks in a semiarid region of south India: implication to sustainable groundwater management. Hum Ecol Risk Assess 25:191-216. https://doi.org/10.1080/10807039.2018.1546550

37. Adimalla N, Dhakate R, Kasarla A, Taloor AK (2020) Appraisal of groundwater quality for drinking and irrigation purposes 
in Central Telangana, India. Groundw Sustain Dev 10:100-334. https://doi.org/10.1016/j.gsd.2020.100334

38. Craig H (1961) Standard for reporting concentrations of deuterium and oxygen-18 in natural waters. Science 133:1833-1834. https://doi.org/10.1126/science.133.3467.1833

39. Mennani A (2001) Apport de l'hydrochimie et de l'isotopie à la connaissance du fonctionnement des aquifères de la zone côtière d'Essaouira (Maroc Occidental). PhD thesis, Cadi Ayyad University, Marrakech, Morocco

40. El Mountassir O, Ouazar D, Bahir M, Chehbouni A, Carreira PM (2021) GIS-based assessment of aquifer vulnerability using DRASTIC model and stable isotope: a case study on Essaouira basin. Arab J Geosci 14:321. https://doi.org/10.1007/ s12517-021-06540-6

41. Clark ID, Frjtz P (1997) Environmental isotopes in hydrogeology. Lewis Publishers, New York

42. Currell MJ, Cartwright I, Bradley DC, Han DM (2010) Recharge history and controls on groundwater quality in the Yuncheng
Basin, north China. J Hydrol 385:216-229. https://doi.org/10. 1016/j.jhydrol.2010.02.022

43. Foster S, Pulido-Bosch A, Vallejos Á, Molina L, Llop A, MacDonald AM (2018) Impact of irrigated agriculture on groundwaterrecharge salinity: a major sustainability concern in semi-arid regions. Hydrogeol J 26:2781-2791. https://doi.org/10.1007/ s10040-018-1830-2

44. Kamal S, Sefiani S, Laftouhi NE, El Mandour A, Moustadraf J, Elgettafi M, Himi M, Casas A (2021) Hydrochemical and isotopic assessment for characterizing groundwater quality and recharge processes under a semi-arid area: case of the Haouz plain aquifer (Central Morocco). J Afr Earth Sci 174:104077. https://doi.org/10.1016/j.jafrearsci.2020.104077

Publisher's Note Springer Nature remains neutral with regard to jurisdictional claims in published maps and institutional affiliations. 